\title{
Gamification Concepts for Leveraging Knowledge Sharing in Industry 4.0
}

\author{
Maria Tsourma $^{1}$, Stylianos Zikos ${ }^{1}$, Georgios Albanis ${ }^{1}$, Konstantinos C. \\ Apostolakis ${ }^{1}$, Evdoxia E. Lithoxoidou ${ }^{1}$, Anastasios Drosou ${ }^{1}$, Dimitrios Zarpalas ${ }^{1}$, \\ Petros Daras ${ }^{1}$, Dimitrios Tzovaras ${ }^{1}$ \\ ${ }^{1}$ Information Technologies Institute, Centre for Research and Technology Hellas, \\ Thessaloniki, Greece \\ \{mtsourma, szikos, galbanis, kapostol, elithoxo, drosou, zarpalas, daras, \\ Dimitrios.Tzovaras\}@iti.gr
}

\begin{abstract}
This paper presents gamification concepts implemented by a gamification engine which is incorporated in a knowledge sharing web-based application. The engine aims at increasing user's motivation and participation in knowledge sharing and training processes taking place on a factory's shop floor, enhance socialization and support corrective feedback and positive reinforcement. In particular, it motivates workers to participate in discussions, propose solutions to work-related problems, and upload/view useful content even when being at the workplace. The gamification engine makes use of various gamification elements and is highly configurable in terms of management of gamified tasks. It is designed to support access by both standard display devices (PCs, tablets, mobile phones) as well as Mixed/Augmented Reality platforms, such as Microsoft HoloLens, which are gaining significant traction with industry verticals. The main novelty of the gamification concepts presented is the ability to utilize dynamic worker profile information which is stored in a central database, in order to improve the effectiveness of the gamified tasks, targeting at more effective usage of the knowledge sharing platform in the Industry 4.0 domain.
\end{abstract}

Keywords: gamification, knowledge sharing, organizational learning, augmented reality, Industry 4.0, industrial environment;

\section{Introduction}

Smart manufacturing processes in Industry 4.0 offer flexibility in production, however the high level of automation results in increased complexity. Industrial work is demanding nowadays, as many skills and high expertise are required in order to operate and monitor modern production lines. In industries, workers may operate different types of machines or have to perform manual tasks that require a specific sequence of actions. In both cases they may have to deal with unexpected events, which require high expertise. As workers or operators of different expertise and experience level may be employed, knowledge sharing is important for solving problems that may arise, or for skills enrichment. To this end, Information Technology (IT) systems such as internal social media platforms are gaining in popularity in such environments. However, a common obstacle in knowledge sharing is the fact that some employees tend to resist sharing their knowledge [1]. Moreover, employees have to be encouraged to use a company's social media platform in a regular basis in order to maintain a live community of engaged workers. Gamification 
pag. 76

can be applied in the context of knowledge sharing among workers in industrial environments, to address the aforementioned issues.

Gamification is defined as the use of gaming elements to improve user experience and user engagement in non-game services and applications [2]. In [3] the authors propose a sequence of activities to make an effective process of gamification, including: identification of the purpose of the gamified task, identification of the transversal objective, selection of supported game mechanics, and analysis of the effectiveness of the implementation.

This paper presents gamification concepts applied in the knowledge sharing domain in Industry 4.0 industrial environments. The goal is to engage workers in knowledge sharing activities through internal social media platforms and across various connected devices, and thus to collect and utilize explicit and tacit knowledge. The main contributions of the present paper are the following:

- Gamification mechanics and elements of a gamification engine are utilized by a webbased Social Media Platform for motivating workers to participate in knowledge sharing. Important features presented include a user-friendly method to create gamified tasks along with an achievement/badge generation approach, which both take into account the industry-related special requirements.

- Integrating these mechanics with Augmented Reality (AR) technology, for facilitating on-site knowledge sharing supported by gamification, and overcoming common problems in the industry [4], such as inadequate worker training, insufficient work experience and the monotony of work.

- Exploitation of profile information about workers/users which is available in Industry 4.0 factories, such as preferences and skills, to support enhanced gamification actions.

The remainder of this paper is structured as follows. In section 2, state-of-art related work about gamification and knowledge sharing is presented. Section 3 introduces the gamification methods that were implemented in a gamification engine. The system and game mechanics for knowledge sharing are described in section 4. Finally, the conclusions are summarized in section 5 .

\section{Related work}

Gamification as a concept has been applied to various domains, such as in education [57], in order to enhance learners' performance, motivation and collaboration. Software engineering is another domain where the use of gamification is gaining ground [8][9]. Moreover, gamification has been utilized in health and fitness applications with the potential to facilitate patient self-management [10-12]. Gamification in the workplace is discussed in [13]. The authors provide examples of where gamification is being used for business improvement or environmental change. Furthermore, in that work implementation of good practices regarding planning, rewards, and visualization, are also provided.

Issues related to the application of gamification to industries have been discussed in recent work. For example, the authors of [14] describe why gamification is important for industry and discuss about the challenges need to be overcome in the introduction of gamification in industrial environments. In [15], the impact of gamification on manufacturing is studied. A smartphone application was designed to gamify Computer Numerical Control (CNC) machines operational jobs. Results showed that the proposed system is capable of enhancing job motivation, job satisfaction, and operational performance. According to study [16], some of the basic requirements of gamification for the production domain are the use of simple visualization elements and information locality. In particular, in order not to distract the user from the work, simple visualization elements must be used and explicit interaction with gamification elements should be 
avoided. Therefore, implicit interaction with gamification elements using motion recognition is ideal for an industrial environment. Moreover, gamification related information must be shown close to the location of the work. As mentioned in [17], the participation in a gamified activity should always happen voluntarily. Therefore, a worker should be able to select if he/she wants to participate in such an activity. The authors of [18] study the acceptance of gamified work processes in the automotive industry. Results showed that the general acceptance of gamification in automotive production is high providing that special requirements are met.

Application of gamification in knowledge sharing in industrial environments has been also studied. For example, the authors in [19] summarize the status of research in the fields of knowledge management and motivational theory and suggest a classification of gamification characteristics with regard to work-related needs and knowledge-exchange barriers. In [20] the authors bridge the concepts of gamification and knowledge sharing to propose an integrative conceptual framework of organizational learning that can be utilized as a reference for gamifying their knowledge sharing practices. Another conceptual framework related to the development of gamified applications in the context of sustainable manufacturing and Industry 4.0 training and education is proposed in [21]. In [22], the authors propose a model for the development of serious games for learning and acquisition of certain competencies in the context of Industry 4.0.

The review of recent publications revealed that many studies present conceptual frameworks describing design principles and methodologies, while a limited number of studies provide actual practical examples for implementing gamification in Industry 4.0. The application of gamification within Industry 4.0 is a novel approach, however, an interesting fact is that gamification is mostly applied in industrial environments for structured training courses, while knowledge sharing for solving problems through discussions among workers has not been considered.

In this paper we provide examples showing how gamification mechanics can be applied to motivate workers to participate in Industry 4.0 knowledge sharing using webbased social media tools and AR devices. The gamification engine which implements the proposed gamification concepts takes into account the requirements of gamification for the production domain that have been mentioned previously in this section. It is userfriendly, does not provide unnecessary information, supports multiple types of gamification elements, as well as voluntary participation of users to gamified tasks.

\section{Gamification methods}

\subsection{Highly customizable design of gamified activities}

The gamification engine utilized in this paper is an extension of the one presented in previous work [23]. This engine supports the implementation of gamified tasks (games) and the definition of teams of users who will be able to participate in a game. The engine developed is highly customizable and supports the parameterization of multiple mechanics, in order to create highly adjustable games. The development of the platform should be based on its use by any person without the need for special programming skills.

The engine supports two distinct user roles, the system's administrator and the standard user. The administrator is responsible for the creation of a gamified task and the customization of the supported elements included in the game definition process. The administrator is also responsible to edit or delete a game. Furthermore, one more role of the administrator concerns the team's formation of standard users, who will be able to take part on a game as a team, through the provided user-interface. On the other hand, standard users are only able to join or leave a game at will. 
pag. 78

A key feature of the gamification engine concerns the creation and definition of gamified tasks, where each gamified task corresponds to a game that represents actual activities that can be performed by workers. For the definition of a gamified task multiple elements are required, where each element has a certain functionality. Those elements are the actions, rules and awards, which all are fully customizable. An action is a task, such as a step in a procedure. Each game may be comprised of more than one action, according to its description and goal. The second element, the rule, associates actions with awards according to the goals of the game. Apart from the aforementioned elements, the gamification engine includes also some other elements, such as the points, levels and badges/achievements. Points are associated directly with actions, as upon the execution of an action, the appropriate number of points is given to the user as a reward for performing the action. Each game is comprised of levels, configured at the game definition process, where the minimum numbers of points that must have been earned in order to promote the user to each level are defined. For further motivation, the use of badges and achievements is introduced. Figure 1 presents the interconnections among the elements required for the definition of a gamified task.

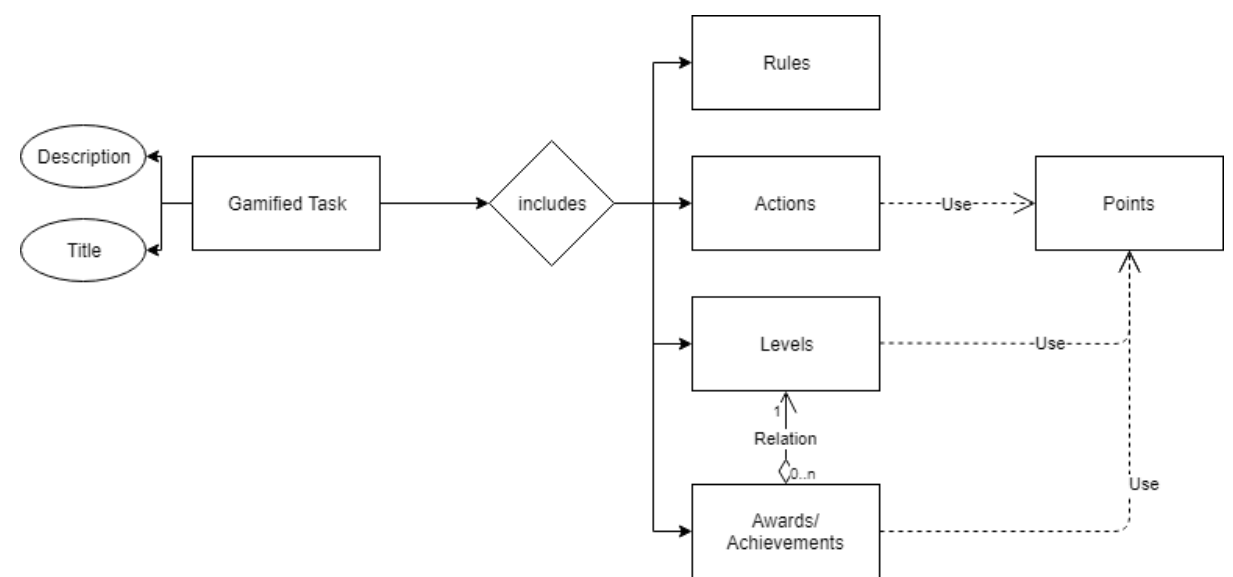

Figure 1. Interconnection among elements required for the definition of a gamified task

For the creation of a gamified task, the system's administrator must perform a certain number of steps to define a game and make it available to the users. The game definition process includes the introduction of a title and a description, because these characteristics must be available to the users. When creating a game, at first the administrator must define the goals of the game and the rules that will be used. As a second step, the actions of the game must be defined and also the point system corresponds to the performance of each action. Additionally, the administrator can define the game levels, awards and badges/achievements and select if the game will be played by individuals or by teams. Figure 2 depicts the process for creating a gamified task as a flowchart.

After a game has been created, its description becomes available. Therefore, the list of games can be retrieved and be shown to a user, who is able to select a game to join in. Similarly, a user can stop his/her participation to a game anytime by selecting to leave the game. The gamification engine involves only the mechanics and operations along with files such as images and shapes which are used for depicting awards and other elements. The visualization part and specific graphical interfaces for displaying elements to the users are separated from the core functionality, allowing to use the gamification engine in different environments.

The gamification engine supports the creation of both individual and team-based gamified tasks. For the creation of teams that will be included in the team-based gamified tasks, the engine supports the creation of several teams, including workers, based on their trade. In this case, one or more groups of users are defined, and awards are given to the whole team and not per individual user. This feature is valuable when gamification is 
applied to industrial environments where employees work in teams and collaborate to achieve a common objective.

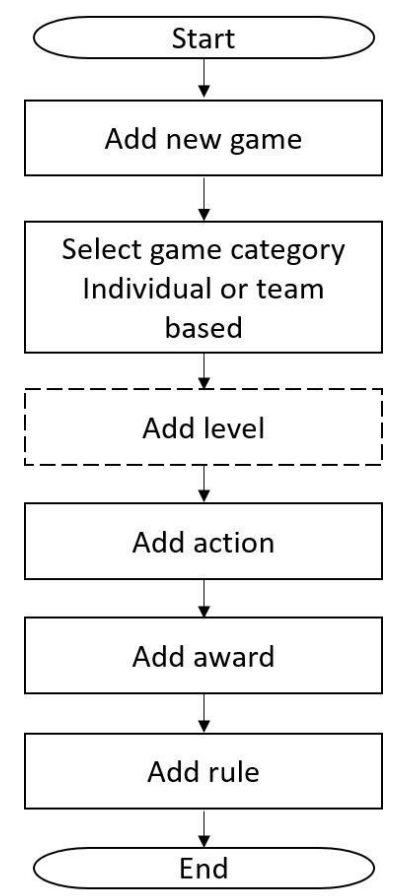

Figure 2. Steps for creating a gamified task. Steps with the dotted outline refer to no mandatory actions for the game definition

\subsection{Tools for Achievements-based gamification}

Badges and achievements are seen as primary mechanics by which services are gamified, and results depict that the motivation of users in using peer-to-peer services is increased [24]. In the video gaming industry, achievements were introduced as a meta-goal, outside of games' parameters, included to extend a game title's longevity and provide players with impetus to do more than simply complete the game. The motivation for the player to gain achievements lies in maximizing their own general cross-title score and obtaining recognition for their performance. A similar game-based thinking and game-based tools can be used in a strategic manner, seamlessly integrating with existing business processes or information systems [25]. Achievements are an ideal mechanic for gamification because they can be implemented without the need to alter or modify the underlying activity [26]. In addition, the philosophy behind achievements is that their difficulty level increases gradually, which will motivate the worker and increase his/her engagement. As defined in [27] an achievement consists of 3 main components: (1) A signifier, (2) completion logic and (3) rewards.

The proposed system implements a web-based Achievement Definition Platform (ADP), which allows users to effortlessly create the previously mentioned components (i.e. signifier-completion logic-rewards) required to define achievements based on the logic described in [27]. Simultaneously, ADP supports auto generation of incremental achievements with an added level of difficulty following the logic lavishly described below. Achievements are usually strictly defined by designers and require very specific conditions to be met in order to be "unlocked". In the system presented the achievements are defined via a new set of common components: (1) an icon, i.e. the unlockable badge, which serves as a signifier; (2) a verb, i.e. what the user does, corresponding to the functional aspect of the completion logic; and (3) an object, a noun representing the object of the verb and corresponds to the quantitative aspect of the completion logic. By specifying these components using a simple form, the platform automatically creates all 
pag. 80

the components of an achievement object by automatically specifying numerical targets, representing the amount of iterations required to "unlock" each achievement. Achievement tiers are therefore automatically generated, in support of incremental difficulty in attaining higher tiers. The ADP automatically creates these milestones for each achievement considering the current tier and based on the following mathematical formula:

where:

$$
\text { new_milestone }=\text { multipliers }[\text { multiplier_idx }] * 100^{i}
$$

- multipliers is a value picked out of the list $[1,5,10,20,50]$.

- multiplier_idx acts as indexing for selecting the proper value in the multipliers array.

- $\quad i \geq 0$ is an integer value that the generator increments by a magnitude of 1 to calculate new_milestone, until the generator finds the first value for which new_milestone > milestone_achieved is satisfied.

Therefore, achievement progression always increment by magnitudes of the multipliers list, e.g. tier 1 requires the user to perform the activity once, tier 2 requires the activity be performed 5 times, and tier 5 requires 50 repetitions. After tier 5 , the $i$ value is incremented by 1 , in which tiers 6 through 10 will require between 100 and 5000 repetitions, and so on.

Table 1 demonstrates the achievement representation format which has been used in the ADP. Figure 3 depicts a screenshot of the ADP, where the auto-definition of elements in Table 1 is clearly visible. In these examples, scores can be added to the achievements to auto-generate more meaningful rewards for workers completing tasks and striving to reach the next tier.

Table 1. Achievement representation format

\begin{tabular}{|c|c|c|}
\hline Component & Generation/Example & Description \\
\hline Icon & icon & $\begin{array}{l}\text { An icon-signifier associated } \\
\text { with each achievement }\end{array}$ \\
\hline Unlock description & $\begin{array}{c}\text { verb }+ \text { target (number) }+ \text { object (plural) } \\
\text { Watch } \underline{\mathbf{1 0}} \text { video } \underline{\mathbf{s}}\end{array}$ & $\begin{array}{l}\text { What the worker has to } \\
\text { do/done in order to unlock the } \\
\text { achievement }\end{array}$ \\
\hline Title & $\begin{array}{c}\text { Object }+\operatorname{verb}(\rightarrow \text { agent noun }) \\
\text { Video watch } \underline{\text { er }}\end{array}$ & $\begin{array}{c}\text { A comprehensive way to } \\
\text { display what activity has to be } \\
\text { carried out in order to unlock } \\
\text { achievement }\end{array}$ \\
\hline Progress & Current iterations / target & $\begin{array}{l}\text { Indicates the worker's progress } \\
\text { for this specific achievement }\end{array}$ \\
\hline
\end{tabular}




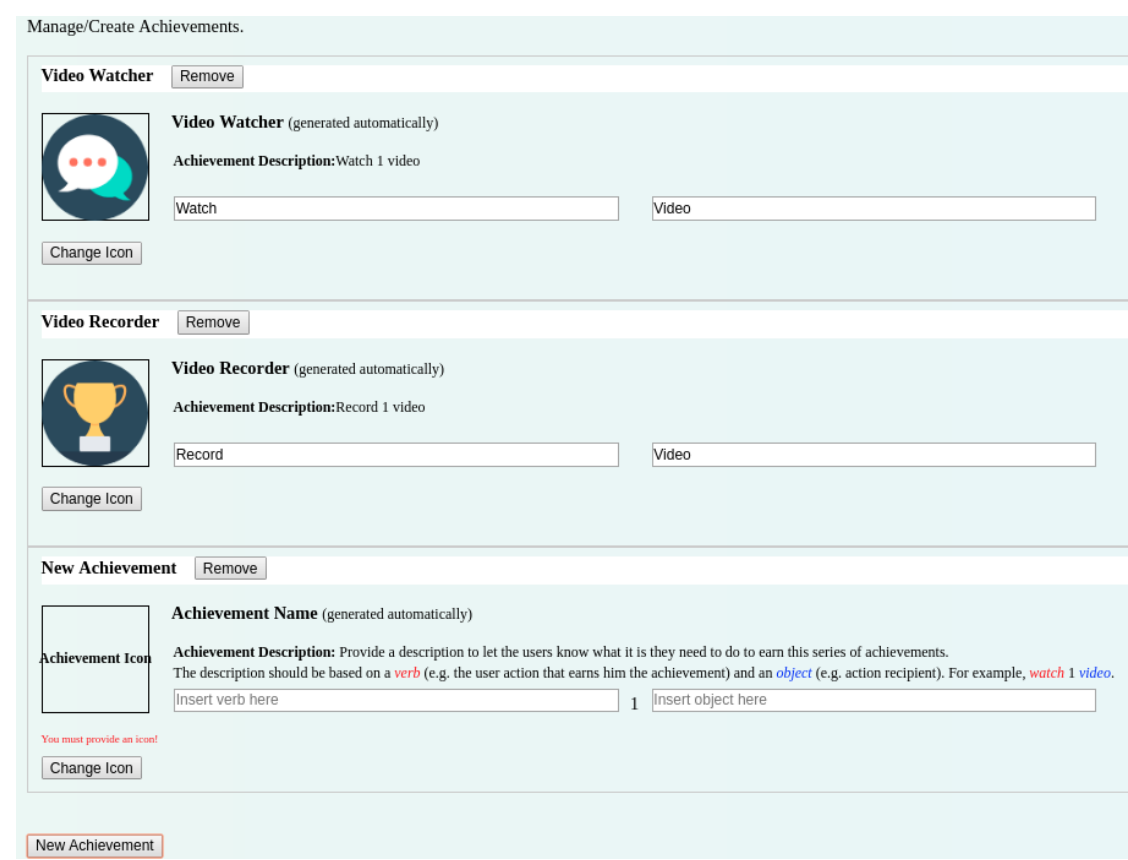

Figure 3. ADP web-based Interface, showcasing minimal user required input based on the icon/verb/object Achievement Representation Format

\section{System description and methodology}

The gamification concepts presented in the previous section have been incorporated into a web-based Social Media Platform which is intended to be used by workers in Industry 4.0 factories, aiming at increasing collaboration and knowledge sharing. In particular, knowledge sharing is important for resolving automation system runtime failures, as multiple automation technologies are usually utilized. Moreover, response times to such incidents must be as short as possible in order not to negatively affect production.

The platform's goal is to increase interaction and socialization among workers, and serve as a repository of tacit knowledge. Through the use of the Social Media Platform, workers are able to upload, view and share multimedia content, exchange opinions with their colleagues, find documentation and guidelines on how to solve problems and ask for help remotely. For supporting these operations, it utilizes various social media technologies such as discussion boards (Figure 5), instant messaging (Figure 6) and news feed with multimedia support. The Social Media Platform has a user login system and supports users of different roles and access rights. Moreover, it allows the user to create a group of co-workers in order to follow their activities towards improving social interaction.

The system architecture is shown in Figure 4. The Social Media Platform is comprised of the back end, which includes the proper REST services for data acquisition and distribution and from a user-friendly user interface (front-end), through which even non-expert users can share knowledge, without any restriction. Apart from all the other functionalities, the user interface displays also all the available games included in the gamification engine and leaderboards showing the user's progress in each game. Alongside, the administrator can manage the gamified tasks and create a new game or a team through the views provided in this user-interface (Figure 7). For privacy reasons, each worker is not allowed to view personal information of other co-workers. Game ranking information retrieved from the gamification engine is based on user rights. Each worker does not have access to view collected points of other co-workers. The main objective is to avoid the negative effects of competition among workers. On the contrary, the system's administrator is able to view the leaderboards of all games. 
pag. 82

Social Media Platform communicates with the Repository central database through REST services for acquiring information about users/workers and multimedia files. It is also able to both retrieve data (e.g. for getting the participants in a game) and send data (e.g. for creating a new gamified activity) to the gamification engine through the engine's REST web services. Augmented reality device communicates with the Social Media Platform for sending and receiving multimedia content and with the gamification engine for notifying about user's actions and retrieving user's scores and achievements.

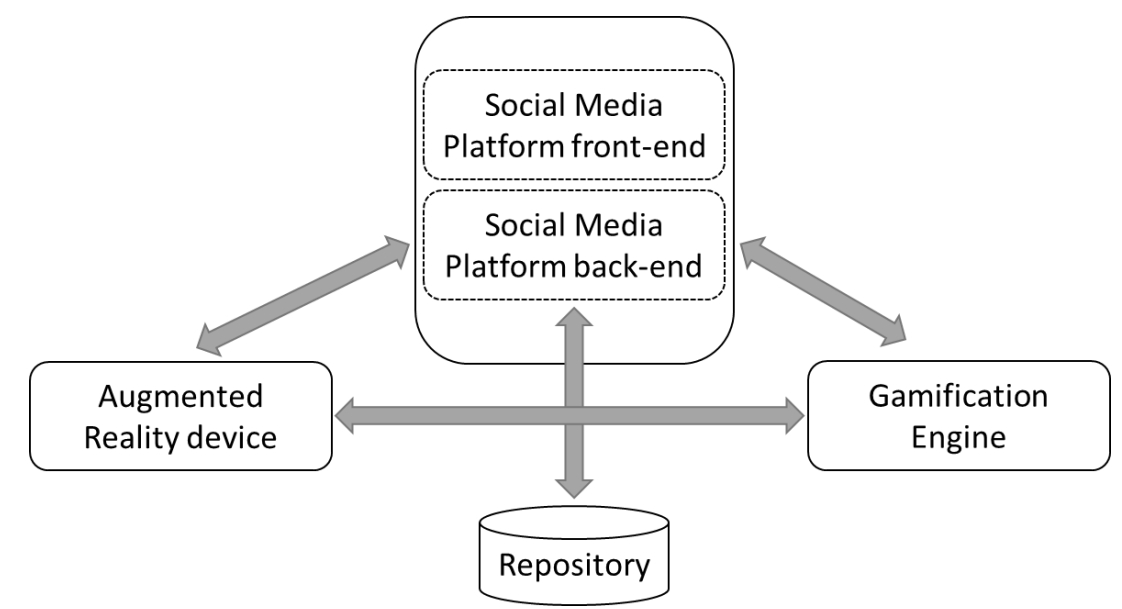

Figure 4. System architecture showing the components involved

\subsection{Social Media Platform Gamification for Industrial Environments}

The gamification engine has the following characteristics regarding its integration with the Social Media Platform:

- Supports multiple users of different types, i.e. administrator and standard users. At least one administrator must be registered for creating gamified tasks.

- Configuration and operation are performed through the use of web services. Therefore, different components are able to interact with the gamification engine in order to retrieve information about a game or user, or update the current status through actions. The gamification engine is also equipped with a data storage mechanism used for storing information regarding games and scores of users in each game, and all the needed information for its proper functionality.

- Notifications can be sent to the user about a new achievement via the Social Media Platform. These are not intrusive in order to not disturb the user when watching content or posting a new discussion topic. The user can navigate to his/her profile page and view the gained achievements on demand.

- Gamification engine can be accessed via diverse mobile devices e.g. tablets and Microsoft HoloLens.

The objective is to create gamified tasks in order to motivate workers (a) to be active members of the Social Media Platform and utilize the stored knowledge and (b) to share their knowledge with other workers through the social media interaction mechanisms that the platform provides (i.e. discussion board). Knowledge sharing is achieved through the discussions board, where users can post or reply to multiple questions regarding issues, such as system failures, they are probably facing at the workplace. A major challenge is to ensure that the content that is uploaded is not inaccurate or poorly-written. As the number of likes a discussion receives is a good indicator for quality, a rule included in a gamified task to give points based on the number of received likes can motivate users to upload useful content of high quality. Furthermore, users are able to watch training videos about automation system error solving or standard operating procedures and can also post videos showing them perform a certain procedure. In this way tacit knowledge can be captured, stored and become available to inexperienced workers. A list of gamified 
actions and their links to the ADP are summarized in Table 2 below. As shown in the table, there are six possible actions through which a user can earn points. The actions 'Upload video', 'Post topic', 'Post reply' and 'Receive like' are related to active participation in knowledge sharing, while the actions 'Watch video' and 'Like comment' are mainly related to the use of the platform.

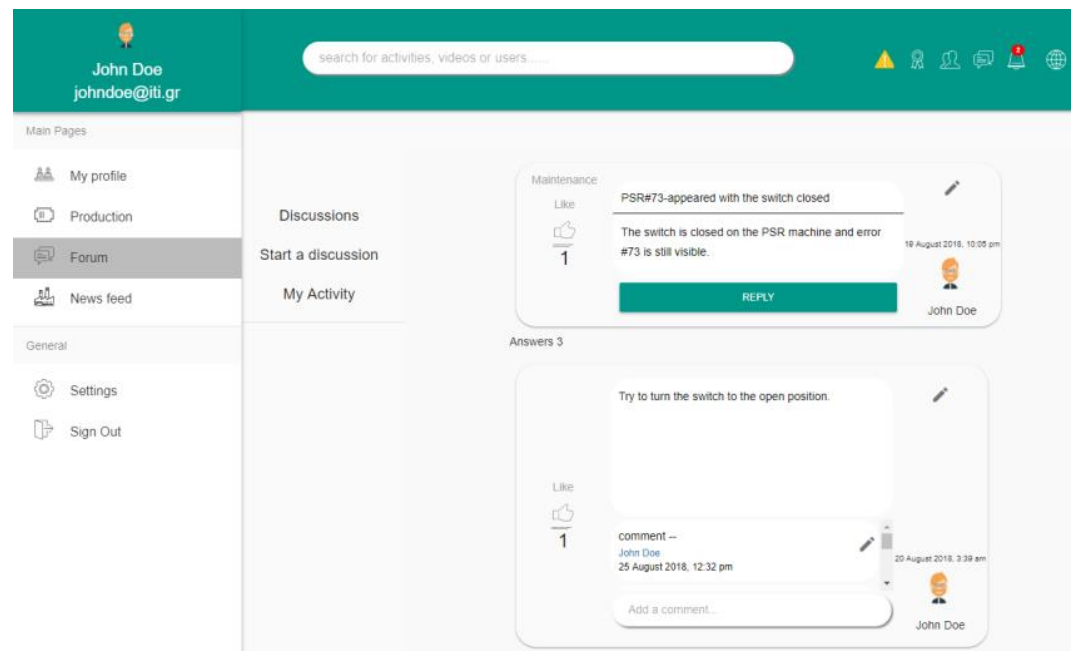

Figure 5. Discussion board as an online forum

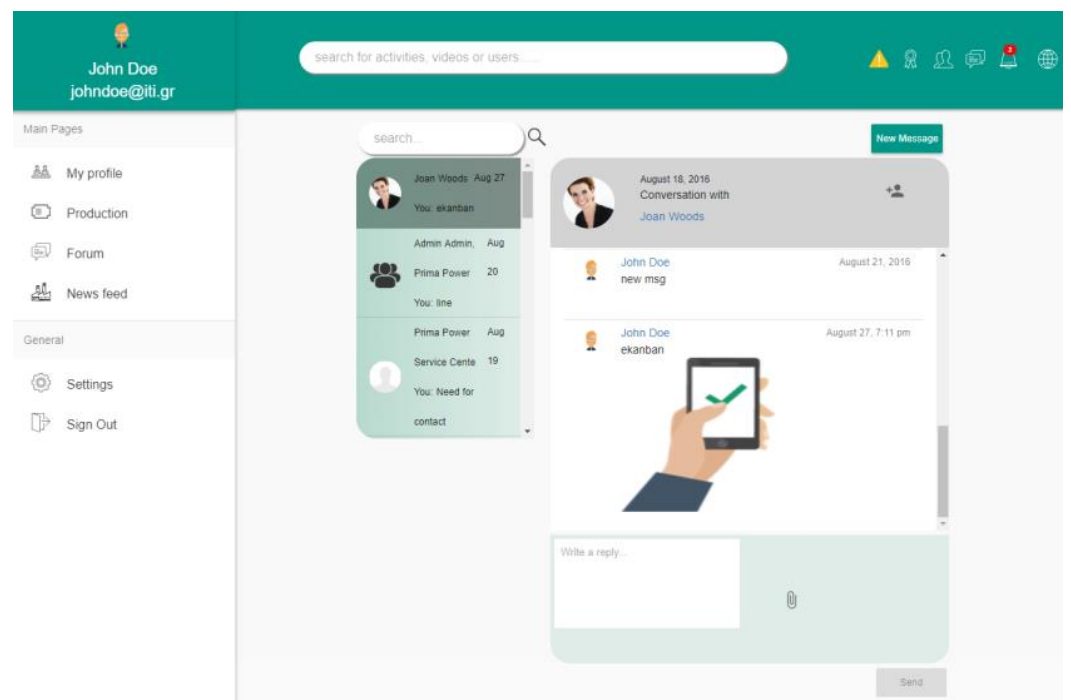

Figure 6. Instant messaging for direct real-time communication

Table 2. Gamified actions and links to ADP

\begin{tabular}{|c|c|}
\hline $\begin{array}{c}\text { Action } \\
\text { Verb }+ \text { Object }\end{array}$ & Description \\
\hline Watch video & The user earns points in case he/she watches a video \\
\hline Upload video & The user earns points in case he/she uploads a new video to the platform \\
\hline Post topic & The user earns points in case he/she posts a new topic in the discussion board \\
\hline Post reply & The user earns points in case he/she posts a reply in the discussion board \\
\hline Like comment & The user earns points in case he/she up votes a comment in the discussion board \\
\hline Receive like & $\begin{array}{l}\text { The user earns points in case his/her comment or post has been up voted by } \\
\text { another user }\end{array}$ \\
\hline
\end{tabular}




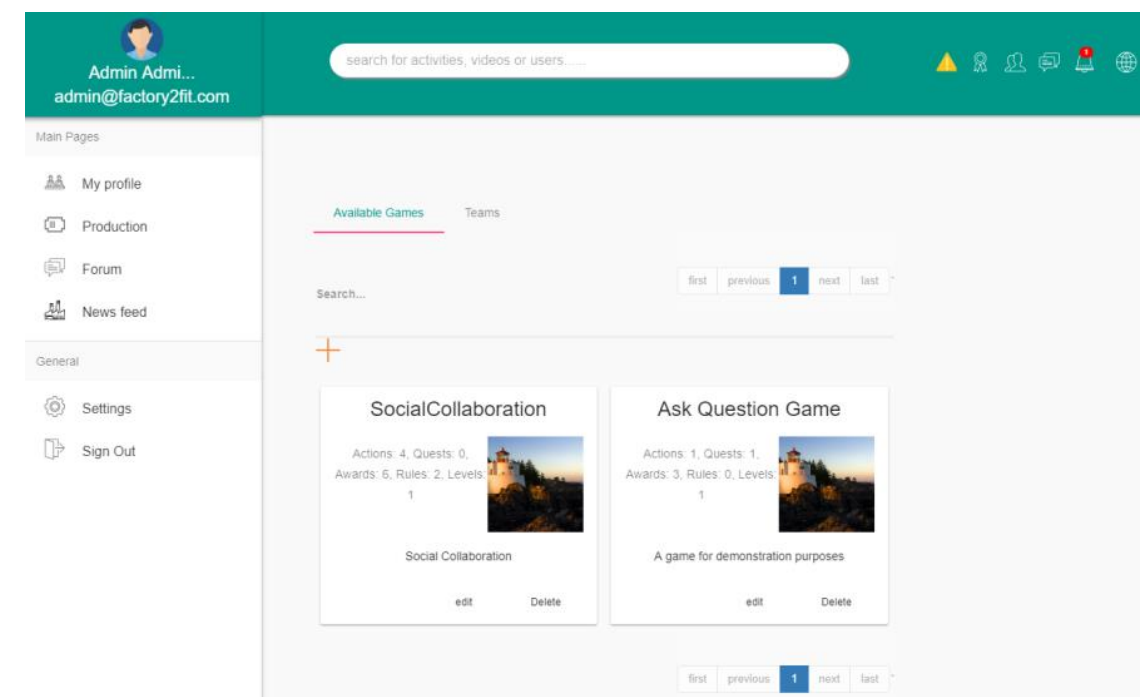

Figure 7. Administrator's view of available games, where a game can be created, edited or deleted

\subsection{Gamification for AR tools}

In general, AR is expected to play an important role in the digital transformation of the factories. The penetration of AR devices in various sectors of industry has increased the last years while hardware limitations have been by passed [28][29]. AR devices are suitable for use at the workplace as they allow employees to use their hands while useful information is displayed in front of them.

To extend functionalities of the gamification engine to the actual activities on the shop floor, an Augmented Gamification Tracker (AGT) as a plug-in interface for augmented reality applications was developed in the Unity Game Engine. The AGT is intended for use in Industry 4.0 industrial environments, where knowledge sharing is facilitated through the Social Media Platform. In addition, AR can be also used for facilitating a Mixed Reality Digital Video Recorder (DVR), through which augmented video content will be created. More specifically, mixed reality videos will be recorded by utilizing the Point of View shots of the device's front-facing camera along with holograms placed by the user to annotate activities. As a next step this video will be uploaded using REST services to the Social Media platform as a knowledge item for other workers to benefit from. Exploiting the modularity of the Social Media platform, AR technology makes it possible for users to upload multimedia content from the AR device to the Social Media Platform and also watch videos or any other useful information to the display provided from the AR device.

The AGT defines achievements as unlockable UI elements, which can be visualized on demand (Figure 8a), by communicating to the user's profile in the social media platform through a REST interface. The plug-in functions through an Achievement Tracker singleton store information for all currently locked and unlocked achievements, as well as progression counters for each individual action. Moreover, "Commendable" behavior scripts are attached to UI events (such as rating or watching a video, earning likes, etc.) which monitors user interactions with the knowledge content, through Social Media Platform, for a specified achievement, and notifies the AGT that the user has been engaged in a commendable activity. Whenever a specified achievement target is reached a notification is sent to the user displayed as an icon on the top right of his/her view on the AR device. Therefore, the user can select the notification icon in order to pop the completed achievement in view and get informed of its unlocked status (Figure 8b). The achievement object is placed in the user's "unlocked" list, while another singleton linked 
to the ADP automatically creates the next progression tier for that specific activity, and places a new achievement object in the user's "locked" achievement's list.

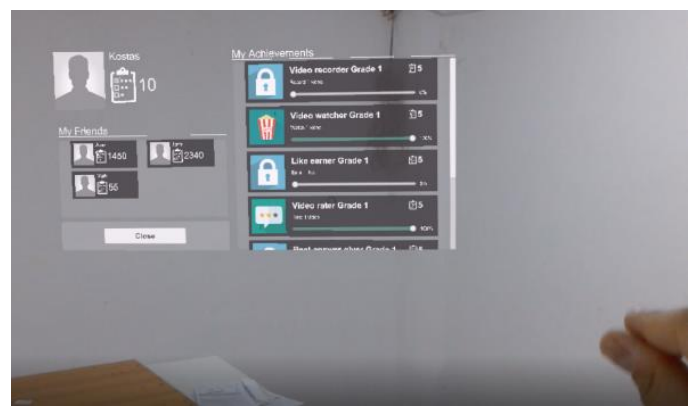

(a)

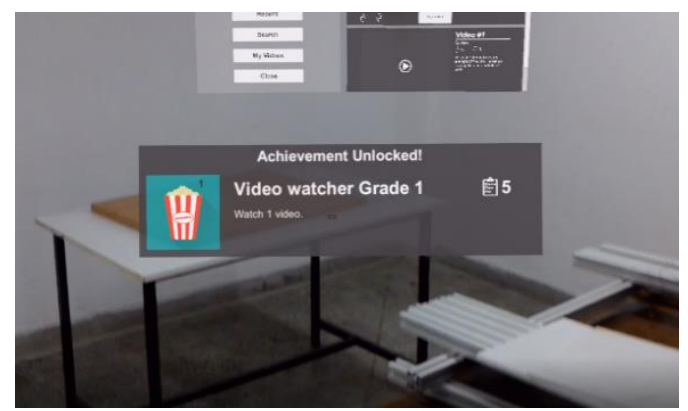

(b)

Figure 8. Screenshots of the Augmented Gamification Tracker, demonstrating: (a) achievement progress and tracking screen; and (b) unlock notification

\subsection{Enhancing gamification using worker profiles}

Since factories employ workers of different competencies and experience, information about workers should be utilized in the gamification mechanics, in order to enhance the gamified tasks and increase their efficiency. Profile information of workers including characteristics such as skills, experience level, and preferences on tasks, co-workers, working shifts, etc., can be available from the factory's database, as personalization is one of the key characteristics in Industry 4.0. Gamification engine can be extended in order to take into account worker profile information, supporting the following scenarios:

- Use of preference settings about most preferred co-workers. Each worker can enter a set of co-workers he/she prefers to work with. This information is used by a Decision Support System when assigning team-based tasks based on multiple criteria. A rule in a gamified task can be created for granting reward points to a worker when he is added to a preference list of a colleague (action).

- Use of skills and experience level. Information such the trade and skills of each worker can be used in order to adapt a game's generic action to a specific user's needs. For example, when the "watch_training_video" action is triggered, additional points can be given in the case the video being watched is relevant to the trade and proportional to the experience level of the worker.

\section{Conclusions}

This paper presented gamification concepts that are suitable for application in Industry 4.0 environments in the context of knowledge sharing. A Social Media Platform that can be used internally in industrial environments and utilizes gamification mechanics has been presented. Through the use of this platform workers can raise discussions for certain issues, such as solutions to automation systems runtime failures, post multimedia content related to training on certain procedures or error solution cases and also reply to other coworker's questions. All these activities have been gamified via the use of a gamification engine whose mechanics were designed in a way that allows them to be used in various contexts. The gamification concepts and mechanics utilized by the Social Media Platform fulfill the requirements for application in industrial environments as these have been documented in the literature, and can be utilized through both standard and Mixed Reality devices installed at the workplace. In addition, the Augmented Gamification Tracker allows the usage of the developed gamification engine in augmented reality applications providing quick and on site access to the users. Leveraging AR users are able to record Mixed Reality videos (i.e. videos with enhanced augmented video content) and 
pag. 86

automatically make them available to the Social Media Platform. Furthermore, gamification concepts which exploit information included in a dynamic worker model are proposed, in order to enhance and increase the efficiency of gamified tasks.

Expected impact of the aforementioned gamification concepts and mechanics on the use of the knowledge sharing platform is to increase motivation of workers to (a) be active users and exploit the stored information in order to make their work trouble-free, (b) share their knowledge through the interaction mechanisms that the platform provides (i.e. discussions, upload videos), and (c) upload useful content of high quality. Furthermore, a gamification-enabled knowledge sharing platform has the potential to improve the quality and quantity of its content as well as worker satisfaction. As a future work, there are plans to implement all the gamification concepts presented and test them in actual industrial environments.

\section{Acknowledgement}

This project has received funding from the European Union's Horizon 2020 research and innovation programme under grant agreement No 723277 (project Factory2Fit). This paper reflects only the authors' view and the Commission is not responsible for any use that may be made of the information it contains.

\section{References}

[1] I. Nonaka, A dynamic theory of organizational knowledge creation, Organization science, Vol. 5, Nr. 1, February 1994. https://doi.org/10.1287/orsc.5.1.14

[2] S. Deterding, M. Sicart, L. Nacke, K. O'Hara, \& D. Dixon, "Gamification. Using game-design elements in non-gaming contexts". In Proceedings of CHI'11 extended abstracts on Human Factors in Computing Systems, ACM, May 2011. https://doi.org/10.1145/1979742.1979575

[3] A. F. Aparicio, F. L. G. Vela, J. L. G. Sánchez, \& J. L. I. Montes, "Analysis and application of gamification". In Proceedings of the Thirteenth International Conference on Interacción Persona-Ordenador, ACM, October 2012. https://doi.org/10.1145/2379636.2379653

[4] R. Masoni, F. Ferrise, M. Bordegoni, M. Gattullo, A. E. Uva, M. Fiorentino, M. Di Donato, Supporting Remote Maintenance in Industry 4.0 Through Augmented Reality, Procedia Manufacturing, Vol. 11, pp. 1296-1302, 2017. https://doi.org/10.1016/j.promfg.2017.07.257

[5] L. Herout, "Application of gamification and game-based learning in education". In Proceedings of the Eighth International Conference on Education and New Learning Technologies, ISBN, 2016. https://doi.org/10.21125/edulearn.2016.1212

[6] D. Dicheva, C. Dichev, G. Agre, G. Angelova, Gamification in Education: A Systematic Mapping Study, The Journal of Educational Technology \& Society, Vol. 18, Nr. 3, 2015.

[7] B. S. Akpolat, \& W. Slany, "Enhancing software engineering student team engagement in a high-intensity extreme programming course using gamification". In Proceedings of the $27^{\text {th }}$ Conference on Software Engineering Education and Training (CSEE\&T), IEEE, April 2014. https://doi.org/10.1109/CSEET.2014.6816792

[8] O. Pedreira, F. García, N. Brisaboa, M. Piattini, Gamification in Software Engineering-A Systematic Mapping, Information and Software Technology, Vol. 57, Elsevier, 2015. https://doi.org/10.1016/j.infsof.2014.08.007

[9] D. Ašeriškis, \& R. Damaševičius, "Gamification of a project management system". In Proceedings of the International Conference on Advances in Computer-Human Interactions, 2014.

[10] A. S. Miller, J. A. Cafazzo, E. Seto, A Game Plan: Gamification Design Principles in mHealth Applications for Chronic Disease Management, Health Informatics Journal, Vol. 22, Nr. 2, SAGE, 2016. https://doi.org/10.1177/1460458214537511

[11]T. Alahäivälä, H. Oinas-Kukkonen, Understanding Persuasion Contexts in Health Gamification: A Systematic Analysis of Gamified Health Behavior Change Support Systems Literature, International Journal of Medical Informatics, Vol. 96, Elsevier, 2016. https://doi.org/10.1016/j.ijmedinf.2016.02.006 
[12]L. Sardi, A. Idri, J. L. Fernández-Alemán, A Systematic Review of Gamification in e-Health, International Journal of Biomedical Informatics, Vol. 71, Elsevier, July 2017. https://doi.org/10.1016/j.jbi.2017.05.011

[13] S. Dale, Gamification: Making Work Fun, or Making Fun of Work?, Business Information Review, Vol. 31, Nr. 2, SAGE, July 2014. https://doi.org/10.1177\%2F0266382114538350

[14] J. Schuldt, \& S. Friedemann, "The challenges of gamification in the age of Industry 4.0: Focusing on man in future machine-driven working environments". In Proceedings of the Global Engineering Education Conference, IEEE, April 2017. https://doi.org/10.1109/EDUCON.2017.7943066

[15] M. Liu, Y. Huang, D. Zhang, Gamification's Impact on Manufacturing: Enhancing Job Motivation, Satisfaction and Operational Performance with Smartphone-based Gamified Job Design, Human Factors and Ergonomics in Manufacturing \& Service Industries, Vol. 28, Nr. 1, Wiley, January 2018. https://doi.org/10.1002/hfm.20723

[16] O. Korn, M. Funk, \& A. Schmidt, "Design approaches for the gamification of production environments: a study focusing on acceptance". In Proceedings of the Eighth ACM International Conference on PErvasive Technologies Related to Assistive Environments (6), ACM, July 2015. https://doi.org/10.1145/2769493.2769549

[17] E. R. Mollick, N. Rothbard, Mandatory Fun: Consent, Gamification and the Impact of Games at Work, The Wharton School Research Paper Series, 30 September 2014. http://dx.doi.org/10.2139/ssrn.2277103

[18] O. Korn, P. Muschick, A. Schmidt, "Gamification of Production? A Study on the Acceptance of Gamified Work Processes in the Automotive Industry". In W. Chung, and C.Shin (Eds.), Advances in Affective and Pleasurable Design. Advances in Intelligent Systems and Computing, vol 483. Springer, Cham, Springer, 2017. https://doi.org/10.1007/978-3-31941661-8_42

[19] T. Wiegand, S. Stieglitz, "Serious fun-effects of gamification on knowledge exchange in enterprises", In E. Plodereder,L.Grunske,E.Schneider and D.Ull, (Eds.), GI-Jahrestagung, 2014.

[20] S. Singhsomransukh, \& D. Heo, "Gamification of knowledge sharing practices: A proposed conceptual framework for organizational learning". In Proceedings of the 14th International Conference on Intellectual Capital Knowledge Management \& Organisational Learning (232), July 2017.

[21]E. Paravizo, O. C. Chaim, D. Braatz, B. Muschard, and H. Rozenfeld, Exploring Gamification to Support Manufacturing Education on Industry 4.0 as an Enabler for Innovation and Sustainability, Procedia Manufacturing, Vol. 21, Elsevier, 2018. https://doi.org/10.1016/j.promfg.2018.02.142

[22] M. Contreras, D. Bonilla, \& A. P. P. Negrón, "Proposal of a model for the development of labor competencies based on serious games in the context of Industry 4.0". In Proceedings of the International Conference on Software Process Improvement, Springer, Cham, October 2018. https://doi.org/10.1007/978-3-030-01171-0_7

[23] E. E. Lithoxoidou, S. Doumpoulakis, A. Tsakiris, S. Krinidis, D. Ioannidis, K. Votis, \& D. Tzovaras, "Improvement of the workers' satisfaction and collaborative spirit through gamification". In Proceedings of International Conference on Internet Science (184), Springer, Cham, 2017. https://doi.org/10.1007/978-3-319-70284-1_15

[24] J. Hamari, Do Badges Increase User Activity? A Field Experiment on the Effects of Gamification, Computers in Human Behavior, Vol. 71, Elsevier, June 2017. https://doi.org/10.1016/j.chb.2015.03.036

[25] A. Mora, D. Riera, C. González, and J. Arnedo-Moreno, Gamification: A Systematic Review of Design Frameworks, Journal of Computing in Higher Education, Vol. 29, Nr. 3, December 2017. https://doi.org/10.1007/s12528-017-9150-4.

[26] G. Goehle, Gamification and web-based homework, Primus, Vol. 23, Nr. 3, March 2013. https://doi.org/10.1080/10511970.2012.736451.

[27] J. Hamari, \& V. Eranti, "Framework for designing and evaluating game achievements". In Proceedings of the Digital Games Research Association International Conference, 2011.

[28] M. A. Frigo, E. C. da Silva, G. F. Barbosa, Augmented Reality in Aerospace Manufacturing: A Review, Journal of Industrial and Intelligent Information, Vol. 4, Nr. 2, March 2016. https://doi.org/10.18178/jiii.4.2.125-130

[29] P. Fraga-Lamas, T. M. Fernández-Caramés, Ó. Blanco-Novoa, M.A. Vilar-Montesinos, A Review on Industrial Augmented Reality Systems for the Industry 4.0 Shipyard, IEEE Access, Vol. 6, 2018. https://doi.org/10.1109/access.2018.2808326 